\title{
MICROFABRICATED QUASIPERIODIC OPTICAL DIFFRACTION APERTURE ARRAYS FOR THE NANOMETROLOGY OPTICAL RULER IMAGING SYSTEM
}

\author{
N. Yoshimizu*, A. Lal, and C. R. Pollock
}

School of Electrical and Computer Engineering, Cornell University, Ithaca NY, 14853

\begin{abstract}
This work demonstrates wafer-scale, path-independent, atomically long term-stable position nanometrology. The system can position multiple objects simultaneously, and could also measure their rotation. This nanometrology optical ruler imaging system uses the diffraction pattern of an atomically stabilized laser from a microfabricated quasiperiodic aperture array as a two dimensional optical ruler. Nanometrology is accomplished by cross correlations of image samples of this optical ruler. This work demonstrates new results showing positioning errors down to 17.2 $\mathrm{nm}$ over wafer scales and long term stability below $20 \mathrm{~nm}$ over six hours. This work also numerically demonstrates the robustness of the optical ruler to variations in the microfabricated aperture array, and discretization noise in imagers.
\end{abstract}

\section{INTRODUCTION}

Tip-based nanofabrication, such as dip-pen writing or thermal surface modification, is a promising technology that will enable future computational and sensor/actuator technologies. In addition, scanning probe microscopy is critical to semiconductor device process characterization and development as future device milestones of sizes down to tens of nanometers. However, their success will rely critically on fast, precise nanometrology of nanoscale features at length scales varying up to seven orders of magnitude. State-of-the-art capacitive sensors and strain sensors lack long travel range and high precision optical encoders require a large reflective block which limits the frequency of movement. The resulting technological roadblock is the inability for a scanning probe microscope to quickly return to the same location after moving distances as small as tens of microns, much less an entire wafer. Consequently, time is laboriously spent searching for nanoscale features, limiting the throughput of nano-science and technology.

The nanometrology optical ruler imaging system (NORIS) uses an atomically stabilized laser as a stable base for metrology. The laser can be stabilized down to parts per billion. Just as atomic clocks use alkali atoms to stabilize a frequency source over long times, here the atoms stabilize the optical source for a longterm stabilized wavelength-determined optical ruler. The wavelength-stabilized laser beam is diffracted by a micropatterned metal thin film, which projects an optical ruler over the wafer-scale workspace and acts as a precise optical ruler. A quasiperiodic pattern is used, which generates sharper features than periodic or randomly generated patterns. Using such a pattern yields much greater precision than using a periodic structure. The system is described elsewhere, but we show a schematic for recall in Figure 1.

A basic idea for NORIS was presented earlier [1]. However, the earlier implementation used a periodic grating aperture array, resulting in a hexagonal lattice diffraction optical ruler. Using a quadrature photodiode, an analogue proportional-integraldifferential control loop was used to position the photodiode on the center of peak in the optical ruler. Marks were made in poly(methyl methacrylate) at points corresponding to the optical ruler. The work resulted in a precision of $\pm 3 \times 10^{-4}$ precision over a $75 \mathrm{~mm}$ wafer.

In this paper, the system has evolved to using a quasiperiodic aperture array which results in a diffraction pattern that has very dense features. This results in great improvement in the precision of NORIS. Preliminary results and the principles of the system are described elsewhere. Here, we numerically demonstrate the robustness of the precision against defects or variations in the microfabricated quasiperiodic aperture array. Finally, this work demonstrates stability of $20 \mathrm{~nm}$ over six hours and precision down to $17 \mathrm{~nm}$.

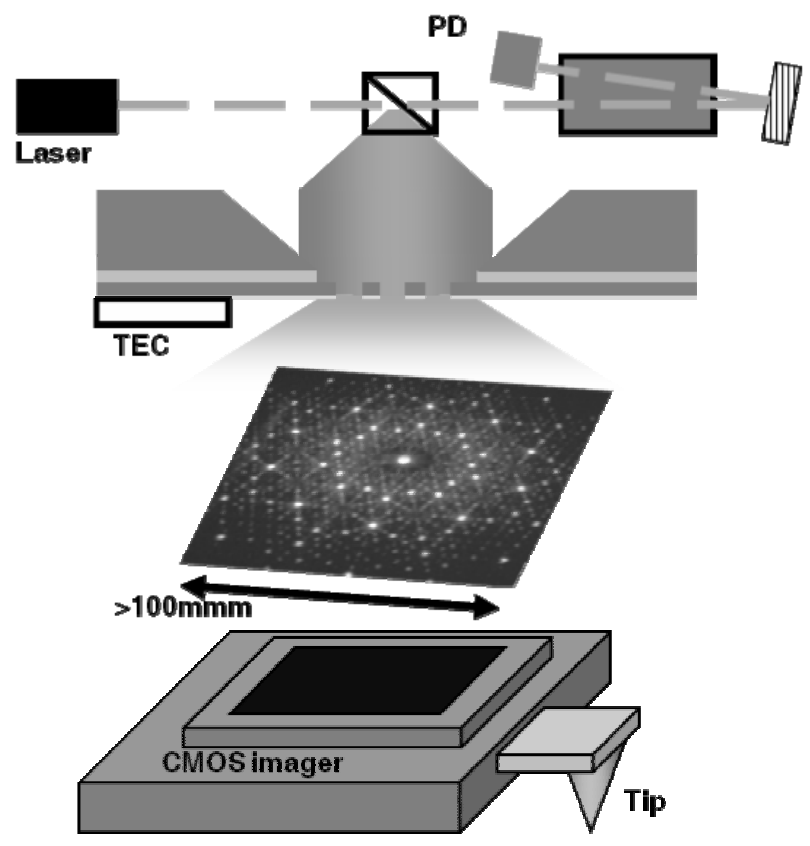

Figure 1: Nanometrology Optical Ruler Imaging System schematic: An external cavity laser is frequency stabilized within 6 $\mathrm{MHz}$, or a relative accuracy of $1.5 \times 10^{-8}$, to a saturated resonance ( $F=2$ to 1 ) of the D2-line of ${ }^{85} \mathrm{Rb} . A 22.77 \pm 0.03{ }^{\circ} \mathrm{C}$ temperature stabilized microfabricated Penrose vertices grating diffracts the laser beam (fabricated using ebeam lithography on SOI device layer; device layer Si etch; thru carrier wafer backside $\mathrm{KOH}$ etch; buffered HF release; Ti/Au evaporation). Wafer-scale optical ruler is shown, and CMOS camera acquired image of a small section of the optical ruler. Tip/CMOS imager is mounted on a commercial stage. Upsampled Fourier transform cross correlation calculates the CMOS imager position within the optical ruler.

\section{QUASIPERIODIC DIFFRACTION}

The optical ruler is generated by the diffraction of an atomically stabilized laser beam by a microfabricated metal thin film. In the far field, or Fraunhofer diffraction region, the amplitude of the diffracted optical field is given by,

$$
\begin{aligned}
U(x, y)=e^{i k z} & e^{i k\left(x^{2}+y^{2}\right) / 2 z} /(i \lambda z) \\
& \times \iint_{ \pm \infty} U(\xi, \eta) \exp [-i 2 \pi(x \xi+y \eta) /(\lambda z)] d \xi d \eta,
\end{aligned}
$$


where the amplitude $\mathrm{U}$ is calculated at position $(x, y, z)$ due to the diffraction of amplitude $U$ at $(\xi, \eta)$ by an optical field of wave vector $k$ and wavelength $\lambda$ [2]. The first factor decreases the optical power density as the optical field diffracts away from its origin. The second factor corresponds to a decrease in the optical field away from its center. The diffraction pattern will have more optical power towards the center of the image, and the optical power will generally decay towards the outer parts of the diffraction pattern. The double integral contains the information for the features in the diffraction pattern, a critical component in achieving high precision in NORIS.

The double integral in Equation (1) is a Fourier transform of the amplitude $\mathrm{U}(\xi, \eta)$ at the diffraction plane. If the amplitude is constant across the aperture array, the double integral is a Fourier transform of the geometric shape of the aperture array where the openings are a constant intensity (normalized to one), and the nontransmitting regions are zero. In order to maximize the precision of the NORIS system, an optical ruler is needed whereby the cross correlation techniques yield the highest precision in the estimates of displacement. As pointed out in [3], the precision can be estimated based on the mean square error of the image registration at some offset $\mathbf{r}$. The lower bound of the mean square error is given by the Cramér-Rao bound,

$$
\operatorname{MSE}(\mathbf{r}) \geq J^{-1}(\mathbf{r}),
$$

assuming an unbiased estimator, where the Fisher information matrix $J$ is,

$$
[J(\mathbf{r})]_{i j}=-E\left[\partial^{2}(\log f) /\left(\partial \mathbf{r}_{i} \partial \mathbf{r}_{j}\right)\right]
$$

which is the negative expectation value of the partial derivatives of the $\log$ of the likelihood function. Here, the likelihood function refers to the image registration, or the calculation of the translational offset of two images.

As might be expected, Equations (2) and (3) show that high precision in image registration will be achieved by using images with image gradients. Most image registration techniques are normalized by intensity to account for lighting or sampling effects, for example. NORIS, which also normalizes its images before the image registration cross correlations, will therefore need image gradients that are different across the whole image so that image registration will see high gradient features of different directions. This will result in high contrast in the cross correlation, resulting in higher image registration precision. Therefore, we are looking for a diffraction array that generates very dense, very sharp features across the optical ruler area. In addition, we would like a diffraction pattern that is translationally asymmetric resulting in unambiguous positioning across a large area.

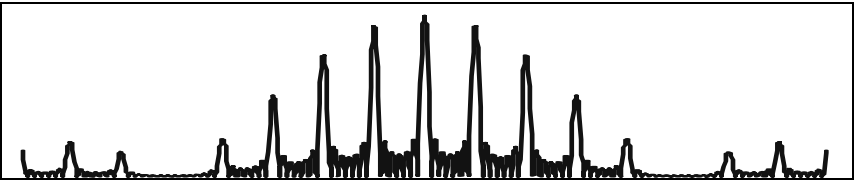

Figure 2: One dimensional diffraction pattern from a seven element, periodic aperture array, estimated by a Fourier transform. The resulting pattern, a sum of sinc functions, results in evenly spaced peaks with an intensity envelope.

An interesting solution can be found by considering different types of periodicity for the diffraction aperture arrays. To simplify the analysis, we consider one-dimensional diffraction, such as in Young's slit experiments. First, consider a very simple one dimensional periodic structure. The screen is opened periodically by some opening of constant width. Therefore, the diffraction plane amplitude $U(\xi, \eta)$ is a periodic array of rect, or unit step, functions. As expected from the readily available analytical solution, the diffraction pattern or Fourier transform of the screen aperture is a shifted sum of sinc functions. The diffraction pattern is a number of peaks with a decaying, periodic envelope of the intensity which decays away from the center of the diffraction pattern. In Figure 2, we show the one dimensional diffraction pattern from a periodic, seven aperture screen. A Fourier transform is used to estimate the resulting diffraction pattern.

Figure 2 shows a number of peaks that are periodic, limited by the bandwidth of the aperture array. However, despite the bandwidth available there are gaps where there is an apparent lack of features. We would like to decrease the mean square error of image registration shown in Equation (1) by increasing the optical gradients across the one dimensional image. As a first attempt, randomness is introduced to the aperture array. Rather than periodically placing the apertures, their locations are randomly shifted. The resulting diffraction pattern is shown in Figure 3. While some smaller intensity structure has appeared, there are number of large peaks that were clearly visible in the periodic case that have completely disappeared. The incoherent shifts in the aperture positions, i.e. white back ground noise, also slightly raises the noise floor of the diffraction pattern resulting in a less signal to noise of the peaks overall.

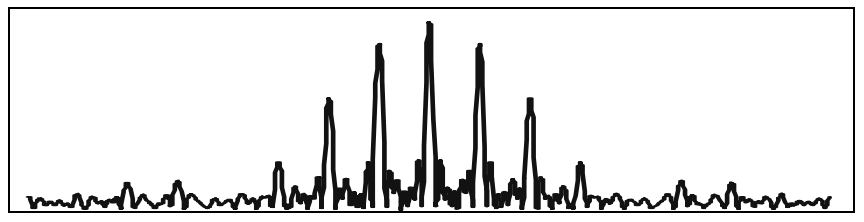

Figure 3: One dimensional diffraction pattern from a seven element, aperiodic aperture array in an attempt to increase features in the diffraction pattern. The aperture positions are nearly periodic, located at slight displacements from their periodic locations in Figure 2; the total bandwidth has even been increase slightly. Some low intensity structure appears to emerge, but some of the larger structures seen in the periodic case have disappeared.

Finally, we consider a quasiperiodic structure. Rather than displacing the periodic structure by random shifts, they are positioned at multiples of an irrational factor. Here, we use the golden ration $\phi=(1+\sqrt{5}) / 2 \approx 1.61803$, and we again give the same amount of bandwidth as the periodic case. The quasiperiodic structure, using an irrational factor, generates a denser diffraction pattern.

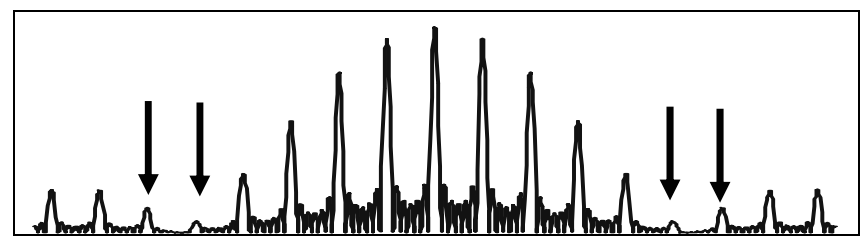

Figure 4: One dimensional diffraction pattern from a seven element, quasiperiodic structure using the irrational Golden Ratio $\phi=(1+\sqrt{5}) / 2 \approx 1.61803 \ldots$ Comparing this diffraction pattern to that in Figure 2, we see that already several new peaks are introduced, marked by arrows. The increase in sharp features increases the precision of NORIS. 
Inituitively, the quaisperiodicy increases the features in the optical diffraction because the irrationality requires more modes in the Fourier transform. Suppose, for example, that the apertures were displaced by a rational shift $m=b / a$. In the Fourier analysis and calculation of the diffraction pattern, each shifted aperture would introduce new components to the diffraction pattern. However, after $a$ number of shifts, the new aperture is shifted by an integer factor $b$ and the new aperture is again in phase with the first aperture. These two apertures belong to the same modes of the Fourier analysis, and no new additional peaks are introduced into the diffraction than would otherwise exist. Quasiperiodicity induced by using irrational numbers, such as the Golden Ratio, results in new components from all the apertures and greatly increases the features in the diffraction pattern. Some of the interesting literature on quasiperiodicity is given in [4-10].

Figure 4 shows the resulting one dimensional diffraction pattern from a quasiperiodic aperture array with equal bandwidth as the periodic case in Figure 2. Although only seven apertures are used, the quasiperiodicity already introduces two new peaks per side in the diffraction pattern, as seen in Figure 4. The introduction of new peaks in the diffraction pattern decreases the mean square error of image registration, as described in Equation (1).

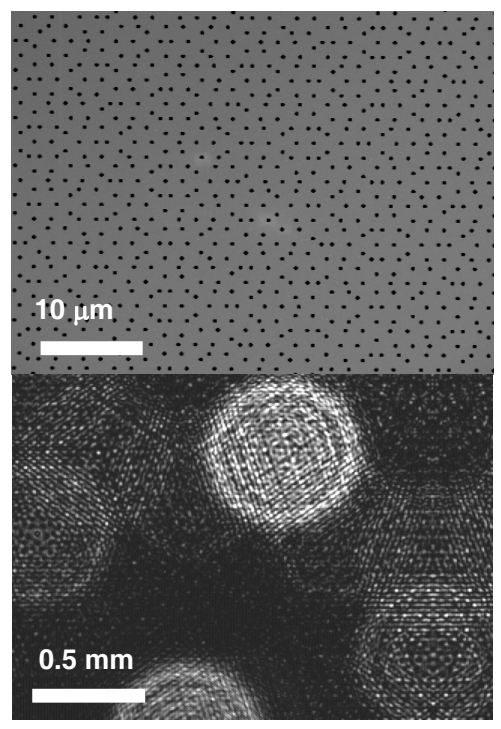

Figure 5: Top: Electron micrograph of a metal thin film quasiperiodic aperture array generated by using the vertices of a Penrose tiling. Bottom: CMOS imager sample of the resulting diffraction pattern. Note the high density of sharp peaks, due to the diffraction from a quasiperiodic structure.

In NORIS, a two-dimensional quasiperiodic structure is required to generate the diffraction optical ruler. We use the quasiperiodic Penrose tiling of the plane using thin and thick rhombuses whereby aperture circles are placed at the vertices of the tiling [11]. The aperture array and the resulting diffraction pattern are shown in Figure 5. Note the high density of peaks in the diffraction pattern, a result of the quasiperiodicity of the location of the apertures.

\section{APERTURE ARRAY PRECISION}

The basis for the stability for NORIS comes from the atomically stable frequency of the laser beam that is diffracted. By using saturation spectroscopy, for example, the laser frequency can be stabilized down to parts per billion which corresponds to nanometers over a six or twelve inch wafer. However, the accuracy of the positioning depends on the accuracy of the optical ruler. The image sampling clearly cannot directly calculate an image registration spanning wafer scale distance, unless the imager itself were that large. Instead, the imagers can be locally positioned accurately to the optical ruler at some position in space and rely on the precise location of that optical feature within that optical ruler. Since the optical ruler diffracts a parts per billion stabilized laser, the stability of the optical wavelength should not be a problem.

The diffractive aperture array, however, can induce errors in the diffraction optical ruler. This is caused by mechanical distortions of the geometry of the aperture array. Mechanical stability data, previously and currently shown in this manuscript, have demonstrated that vibrational and temperature fluctuation effects are insignificant. For example, the aperture array is a thermally conductive thin film metal spanning only a millimeter. A large thermoelectric cooler can easily stabilize the temperature of such a small thermal load to within $0.03{ }^{\circ} \mathrm{C}$. With thermal coefficients of expansion of parts per million and rigid support around structure, dynamic thermal changes are expected to remain in the parts or many parts per billion as required by NORIS.

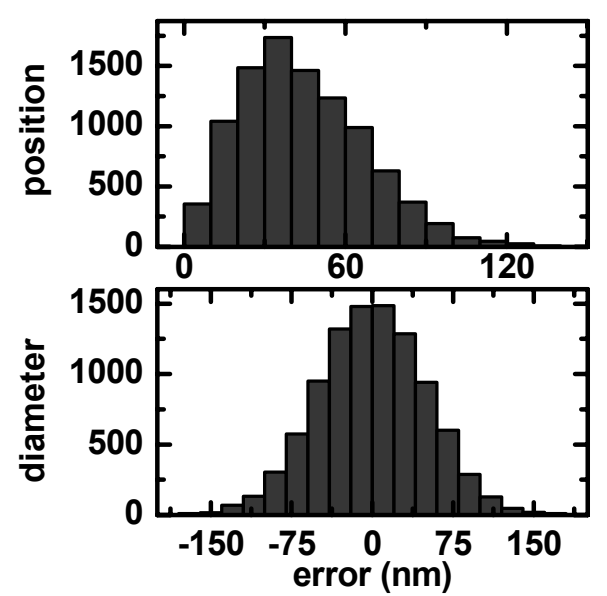

Figure 6: Histogram of aperture diameter and positioning errors, for simulated errors of $50 \mathrm{~nm}$ in microfabrication.

There is, however, a dependency on the resulting diffraction optical ruler due to errors in the actual microfabrication of the aperture array. There are possible sources for microfabrication errors in the aperture array. The size of the holes are nonuniform for slight variations in etching rates and aspect ratios, mask exposure and development, etc. However, the diffraction pattern is a result of the sum of the amplitudes from all apertures which is expected to dilute the effect of small variations in the microfabrication of the aperture array. In addition, as discussed in the section above, the Fourier transform of incoherent changes in the aperture array will tend to add white noise to the diffraction pattern, i.e. raise the noise floor, rather than change the peak structures within the optical ruler.

To characterize the dependence of NORIS precision on variations in the aperture array, numerical simulations were carried out. A $780 \mathrm{~nm}$ optical plane wave was simulated to illuminate an aperture array of 9662, $3 \mu \mathrm{m}$ holes spanning a millimeter in size placed at the vertices of a Penrose tiling, with a $10 \mu \mathrm{m}$ length constant. This is the configuration shown in the electron micrograph in Figure 5. The Fruanhofer diffraction at a distance of $25.4 \mathrm{~mm}$ is calculated, and the resulting optical ruler used to 
calculate NORIS positioning. The aperture array is varied by both the position of the apertures and the diameter of the apertures, using a randomly generated normalized distribution.

The aperture arrays were varied by their diameter and position offset of 20, 50, 100 and $500 \mathrm{~nm}$; a sample histogram for $50 \mathrm{~nm}$ offset is shown in Figure 6.

The resulting position errors are shown in Figure 7, using a $500 \times 500,1 \mu \mathrm{m}$ pixel imager and displaced in $\mathrm{x}$ from 0 to $200 \mu \mathrm{m}$. Error is added to the aperture array, then the optical ruler is image registered to the image from a zero error aperture array. Microfabrication errors up to $200 \mathrm{~nm}$ appear to have little effect on the positioning, but microfabrication errors of $500 \mathrm{~nm}$ have a noticeable effect on NORIS precision. The quasiperiodic diffraction optical ruler appears to be robust to tolerance in microfabrication.

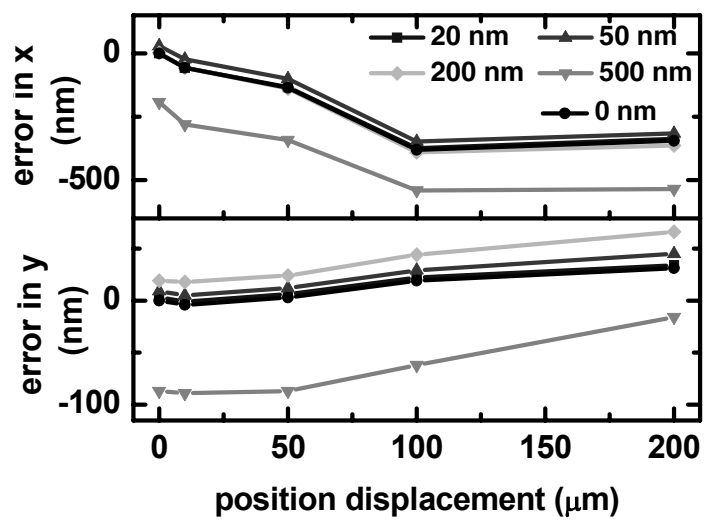

Figure 7: NORIS positioning errors due to microfabrication errors. Positions are calculated by positioning NORIS based on image registration to error aperture array to zero error aperture array. Errors of up to $200 \mathrm{~nm}$ appear to have no impact on positioning error; a $500 \mathrm{~nm}$ fabrication error shows an effect on NORIS positioning error.

\section{EXPERIMENTAL RESULTS}

Finally, we show empirical results of NORIS. Figure 8 shows NORIS positioning compared to a short range, high precision capacitive sensor on a piezoelectric flexural stage (nPoint NPXYZ100B), with $17.2 \mathrm{~nm}$ mean absolute deviation. The inset shows the residual. Figure 9 shows the stability of NORIS over 6 hours, at less than $20 \mathrm{~nm}$.

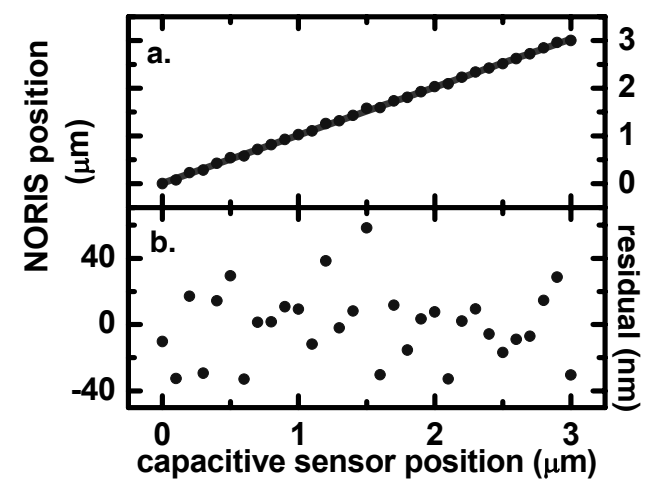

Figure 8: NORIS positioning errors across $3 \mu \mathrm{m}$ displacement, compared to short range, high precision capacitive sensors in a piezoelectric flexural stage.

\section{CONCLUSION}

We have demonstrated the high precision and stability of NORIS, a system for parallel, path-independent, wafer-scale high precision nanometrology for scanning probe microscopes and nanoresolution stages. We have also demonstrated the robustness of the system against microfabrication variations in its quasiperiodic aperture array.

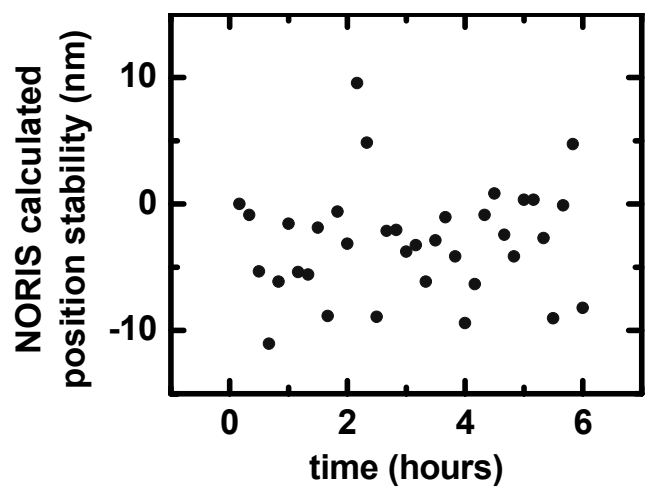

Figure 9: NORIS positioning errors across 3 m displacement.

\section{ACKNOWLEDGEMENTS}

This work was generously funded by the DARPA/TBN program and performed, in part, at the Cornell NanoScale Facility.

\section{REFERENCES}

[1] N. Yoshimizu, A. Lal, C. R. Pollock, "MEMS Diffractive Optical Nanoruler Technology for Tip-Based Nanofabrication and Metrology," Proceedings of the IEEE MEMS 2009, pp. 547-550.

[2] J. W. Goodman, "Introduction to Fourier Optics," Roberts \& Company Publishers Colorado, 2005.

[3] D. Robinson, P. Milanfar, "Fundamental Performance Limits in Image Registration," IEEE Trans. Image. Process 13, pp 1185-11992004.

[4] R. K. P. Zia, W. J. Dallas, "A Simple Derivation of QuasiCrystalline Spectra," J. Phys. A: Math. Gen. 18, pp. L341L345 1985.

[5] V. Elser, "The Diffraction of Projected Structures," Acta. Cryst. A42, pp. 36-43 1986.

[6] F. Gähler, J. Rhyner, "Equivalence of the Generalized Grid and Projection Methods for the Construction of Quasiperiodic Tilings,” J. Pys. A.: Math. Gen. 19, pp. 267-277 1986.

[7] D. Levine, P. J. Steinhardt, "Quasicrystals I. Definition and Structure,” Phys. Rev. B 34, pp 596 - 6161986.

[8] J. E. S. Socolar, P. J. Steinhardt, "Quasicrystals II. Unit-cell Configurations," Phys. Rev. B 617 - 6471986.

[9] C. Janot, "Quasicrystals: A Primer," Oxford University Press, 1997.

[10] M. Senechal, "Quasicrystals and Geometry," Cambridge University Press, 1995.

[11] N. G. de Bruijn, "Algebraic theory of Penrose's non-periodic tilings of the plane I \& II," Ned. Akad. Wetensch. Proc. Ser. A 43, pp 39-66, 1981.

[12] N. Yoshimizu, A. Lal, C. R. Pollock, "Nanometrology Using a Quasiperiodic Pattern Diffraction Optical Ruler," to be published in IEEE JMEMS.

\section{CONTACT}

*N. Yoshimizu, tel: +1-925-998-3299; ny22@cornell.edu 